\title{
Learning the Structure of Objects from Web Supervision
}

\author{
David Novotny ${ }^{1,2(凶)}$, Diane Larlus ${ }^{2}$, and Andrea Vedaldi ${ }^{1}$ \\ 1 Visual Geometry Group, University of Oxford, Oxford, UK \\ \{david, vedaldi\}@robots.ox.ac.uk \\ ${ }^{2}$ Computer Vision Group, Xerox Research Centre Europe, Meylan, France \\ diane.larlus@xrce.xerox.com
}

\begin{abstract}
While recent research in image understanding has often focused on recognizing more types of objects, understanding more about the objects is just as important. Learning about object parts and their geometric relationships has been extensively studied before, yet learning large space of such concepts remains elusive due to the high cost of collecting detailed object annotations for supervision. The key contribution of this paper is an algorithm to learn geometric and semantic structure of objects and their semantic parts automatically, from images obtained by querying the Web. We propose a novel embedding space where geometric relationships are induced in a soft manner by a rich set of non-semantic mid-level anchors, bridging the gap between semantic and non-semantic parts. We also show that the resulting embedding provides a visually-intuitive mechanism to navigate the learned concepts and their corresponding images.
\end{abstract}

Keywords: Object part detection $\cdot$ Web supervision $\cdot$ Mid-level patches

\section{Introduction}

Modern deep learning methods have dramatically improved the performance of computer vision algorithms, from image classification [1] to image captioning [2,3] and activity recognition [4]. Even so, image understanding remains rather crude, oblivious to most of the nuances of real world images. Consider for example the notion of object category, which is a basic unit of understanding in computer vision. Modern benchmarks consider an increasingly large number of such categories, from thousands in the ILSVRC challenge [5] to hundred thousands in the full ImageNet [6]. Despite this ontological richness, there is only limited understanding of the internal geometric structure and semantics of these categories.

Electronic supplementary material The online version of this chapter (doi:10. 1007/978-3-319-49409-8_19) contains supplementary material, which is available to authorized users. 


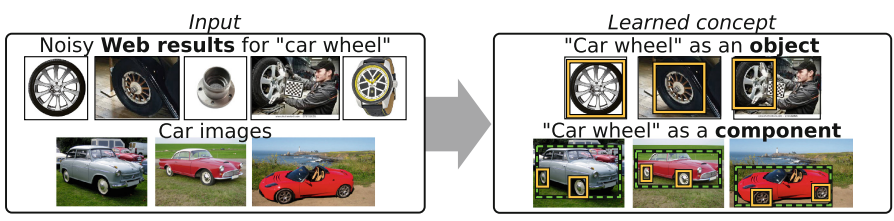

Fig. 1. Our goal is to learn the semantic structure of objects automatically using Web supervision. For example, given noisy images obtained by querying an Internet search engine for "car wheel" and for "cars", we aim at learning the "car wheel" concept, and its dual nature: as an object in its own right, and as a component of another object.

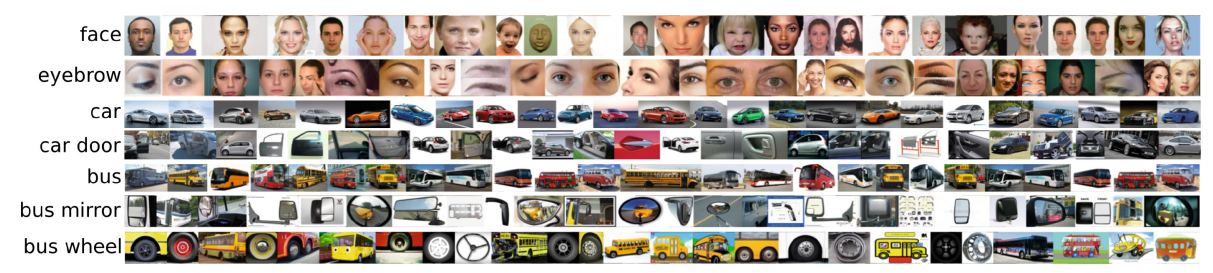

Fig. 2. Top images retrieved from an Internet search engine for some example queries. Note that part results are more noisy than full object results (the remaining collected images get even noisier, not shown here).

In this paper we aim at learning the internal details of object categories by jointly learning about objects, their semantic parts, and their geometric relationships. Learning about semantic nameable parts plays a crucial role in visual understanding. However, standard supervised approaches are difficult to apply to this problem due to the cost of collecting large quantities of annotated example images. A scalable approach needs to discover this information with minimal or no supervision.

As a scalable source of data, we look at Web supervision to learn the structure of objects from thousands of images obtained automatically by querying search engines (crf. Fig. 1). This poses two significant challenges: identifying images of the semantic parts in very noisy Web results (crf. Fig. 2) while, at the same time, discovering their geometric relationships. The latter is particularly difficult due to the drastic scale changes of parts when they are imaged in the context of the whole object or in isolation.

If parts are looked at independently, noise and scale changes can easily confuse image recognition models. Instead, one needs to account for the fact that object classes have a well-defined geometric structure which constraints how different parts fit together. Thus, we need to introduce a geometric frame that can, for any view of an object class, constrain and regularize the location of the visible semantic parts, establishing better correspondences between views.

Traditional representations such as spring models were found to be too fragile to work in our Webly-supervised setting. To solve this issue, we introduce a novel vector embedding that encodes the geometry of parts relatively to a robust 
reference frame. This reference frame builds on non-semantic anchor parts which are learned automatically using a new method for non-semantic part discovery (Sect. 2.2); we show that this method is significantly better than alternative and more complex techniques for part discovery. The new geometric embedding is further combined with appearance cues and used to improve the performance in semantic part detection and matching.

A byproduct of our method is a large collection of images annotated with objects, semantic parts, and their geometric relationships, that we refer to as a visual semantic atlas (Sect.4). This atlas allows to visually navigate images based on conceptual and geometric relations. It also emphasizes the dual nature of parts, as components of an object and as semantic categories, by naturally bridging images that zooms on a part or that contain the object as a whole.

\subsection{Related Work}

Our work touches on several active research areas: localizing objects with weak supervision, learning with Web images, and discovering or learning mid-level features and object parts.

Localizing Objects with Weak Supervision. When training models to localize objects or parts, it is impractical to expect large quantities of bounding box annotations. Recent works have tackled the localization problem with only image-level annotations. Among them, weakly supervised object localization methods [7-13] assume for each image a list of every object type it contains. In the co-detection [14-17] and co-segmentation [18-21] problems, the algorithm is given a set of images that all contain at least one instance of a particular object. They differ in their output: co-detection predicts bounding boxes, while segmentation predicts pixel-level masks. Yet, co-detection, co-segmentation and weaklysupervised object localization (WSOL) are different flavors of the localization problem with weak supervision. For co-detection and WSOL, the task is nearly always formulated as a multiple instance learning (MIL) problem [7, 8, 16, 22, 23]. The formulation in $[11,12]$ departs from MIL by leveraging the strong annotations for some categories to transfer knowledge to the remaining categories. A few approaches model images using topic models [10,17].

Recently, CNN architectures were also proved to work well in weakly supervised scenarios [24]. We will compare with [24] in the experiments section. None of these works have considered semantic parts. Closer to our work, the method of [25] proposes unsupervised discovery of dominant objects using part-based region matching. Because of its unsupervised process, this method is not suited to name the discovered objects or matched regions, and hence lack semantics. Yet we also compare with this approach in our experiments.

Learning from Web Supervision. Most previous works [26-29] that learn from noisy Web images have focused on image classification. Usually, they adopt an iterative approach that jointly learns models and finds clean examples of a target concept. Only few works have looked at the problem of localization. Some approaches $[21,30]$ discover common segments within a large set of Web images, 
but they do not quantitatively evaluate localization. The recent method of [31] localizes objects with bounding boxes, and evaluate the learnt models, but as the previous two, it does not consider object parts.

Closer to our work, [32] aims at discovering common sense knowledge relations between object categories from Web images, some of which correspond to the "part-of" relation. In the process of organizing the different appearance variations of Webly mined concepts, [33] uses a "vocabulary of variance" that may include part names, but those are not associated to any geometry.

Unsupervised Parts, Mid-level Features, and Semantic Parts. Objects are modeled using the notion of parts since the early work on pictorial structure [34], in the constellation [35] and ISM [36] models, and more recently the DPM [37]. Parts are most commonly defined as localized components with consistent appearance and geometry in an object. All these works have in common to discover object parts without naming them. In practice, only some of these parts have an actual semantic interpretation. Mid-level features [38-43] are discriminative [41,44] or rare [38] blocks, which are leveraged for object recognition. Again, these parts lack semantic. The non-semantic anchors that we use share similarities with [45] and [42], that we discuss in Sect.2.2. Semantic parts have triggered recent interest [46-48]. These works require strong annotations in the form of bounding boxes [46] or segmentation masks [47,48] at the part level. Here we depart from existing work and aim at mining semantic nameable parts with as little supervision as possible.

\section{Method}

This section introduces our method to learn semantic parts using weak supervision from Web sources. The key challenge is that search engines, when queried for object parts, return many outliers containing other parts as well, the whole object, or entirely unrelated things (Fig. 2). In this setting, standard weaklysupervised detection approaches fail (Sect.3). Our solution is a novel, robust, and flexible representation of object parts (Sect. 2.1) that uses the output of a simple but very effective non-semantic part discovery algorithm (Sect. 2.2).

\subsection{Learning Semantic Parts Using Non-semantic Anchors}

In this section, we first flesh out our method for weakly-supervised part learning and then dive into the theoretical justification of our choices.

MIL: Baseline, Context, and Geometry-Aware. As standard in weaklysupervised object detection, our method starts from the Multiple Instance Learning (MIL) [49] algorithm. Let $\mathbf{x}_{i}$ be an image and let $\mathcal{R}\left(\mathbf{x}_{i}\right)$ be a shortlist of image regions $R$ that are likely to contain objects or parts, obtained for instance using selective search [50]. Each image $\mathbf{x}_{i}$ can be either positive $y_{i}=+1$ if it is deemed to contain a certain part or negative $y_{i}=-1$ if not. MIL fits to this 
data a (linear) scoring function $\left\langle\phi\left(\mathbf{x}_{i} \mid R\right), \mathbf{w}\right\rangle$, where $\mathbf{w}$ is a vector of parameters and $\phi\left(\mathbf{x}_{i} \mid R\right) \in \mathbb{R}^{d}$ is a descriptor of the region $R$ of image $\mathbf{x}_{i}$, by minimizing:

$$
\min _{\mathbf{w} \in \mathbb{R}^{d}} \frac{\lambda}{2}\|\mathbf{w}\|^{2}+\frac{1}{n} \sum_{i=1}^{n} \max \left\{0,1-y_{i} \max _{R \in \mathcal{R}\left(\mathbf{x}_{i}\right)}\left\langle\phi\left(\mathbf{x}_{i} \mid R\right), \mathbf{w}\right\rangle\right\}
$$

In practice, Eq. (1) is optimized by alternatively selecting the maximum scoring region for each image (also known as "re-localization") and optimizing w for a fixed selection of the regions. In this manner, MIL should automatically discover regions that are most predictive of a given label, and which therefore should correspond to the sought visual entity (object or semantic part). However, this process may fail if descriptors are not sufficiently strong.

For baseline MIL the descriptor $\phi(\mathbf{x} \mid R)=\phi^{a}(\mathbf{x} \mid R) \in \mathbb{R}^{d_{a}}$ captures the region's appearance. A common improvement is to extend this descriptor with context information by appending a descriptor of a region $R^{\prime}=\mu(R)$ surrounding $R$, where $\mu(R)$ isotropically enlarges $R$; thus in context-aware MIL, $\phi(\mathbf{x} \mid R)=\operatorname{stack}\left(\phi^{a}(\mathbf{x} \mid R), \phi^{a}(\mathbf{x} \mid \mu(R))\right)$.

Neither baseline or context-aware MIL leverage the fact that objects have a well-defined geometric structure, which significantly constrains the search space for parts. DPM uses such constraints, but as a fixed set of geometric relationships between part pairs that are difficult to learn when examples are extremely noisy. Furthermore, DPM-like approaches learn the most visually-stable parts, which often are not the semantic ones.

We propose here an alternative method that captures geometry indirectly, on top of a rich set of unsupervised mid-level non-semantic parts $\left\{p_{1}, \ldots, p_{K}\right\}$, which we call anchors (Fig. 3). Let us assume that, given an image $\mathbf{x}$, we can locate the (selective search) regions $R_{p_{k}, \mathbf{x}}$ containing each anchor $p_{k}$. We define the following geometric embedding $\phi^{g}$ of a region $R$ with respect to the anchors:

$$
\phi^{g}(\mathbf{x} \mid R)=\left[\begin{array}{c}
\rho\left(R, R_{p_{1}, \mathbf{x}}\right) \\
\vdots \\
\rho\left(R, R_{p_{K}, \mathbf{x}}\right)
\end{array}\right] .
$$

Here $\rho$ is a measure such as intersection-over-union (IoU) that tells whether two regions overlap. By choosing a function $\rho$ such as IoU which is invariant to

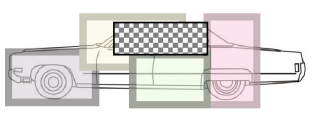

(a)

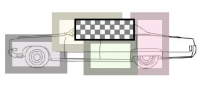

(b)

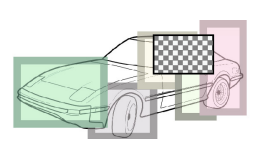

(c)

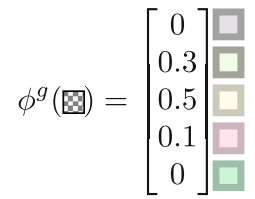

Fig. 3. Anchor-induced geometry. (a) A set of anchors (light boxes) are obtained from a large number of unsupervised non-semantic part detectors. The geometry of a semantic part or object is then expressed as a vector $\phi^{g}$ of anchor overlaps. (b) The representation is scale and translation invariant. (c) The representation implicitly codes for multiple aspects. 
scaling, rotation, and translation of the regions, so is the embedding $\phi^{g}$. Hence, as long as anchors stay attached to the object, $\phi^{g}(\mathbf{x} \mid R)$ encodes the location of $R$ relative to an object-centric frame. This representation is robust because, even if some anchors are missing or misplaced, the vector $\phi^{g}(\mathbf{x} \mid R)$ is not greatly affected. The geometric encoding $\phi^{g}(\mathbf{x} \mid R)$ is combined with the appearance descriptor $\phi^{a}(\mathbf{x} \mid R)$ in a joint appearance-geometric embedding

$$
\phi^{a g}(\mathbf{x} \mid R)=\phi^{a}(\mathbf{x} \mid R) \otimes \phi^{g}(\mathbf{x} \mid R)
$$

where $\otimes$ is the Kronecker product. After vectorization, this vector is used as a descriptor $\phi(\mathbf{x} \mid R)=\phi^{a g}(\mathbf{x} \mid R)$ of region $R$ in geometry-aware MIL. The next few paragraphs discuss its properties.

Modeling Multiple Parts. Plugging $\phi^{a g}$ of Eq. (3) into Eq. (1) of MIL results in the scoring function $\left\langle\mathbf{w}, \phi^{a g}(\mathbf{x} \mid R)\right\rangle=\sum_{k=1}^{K}\left\langle\mathbf{w}_{k}, \phi^{a}(\mathbf{x} \mid R)\right\rangle \rho\left(R, R_{p_{k}, \mathbf{x}}\right)$ which interpolates between $K$ appearance models based on how the region $R$ is geometrically related to the anchors $R_{p_{k}, \mathbf{x}}$. In particular, by selecting different anchors this model may capture simultaneously the appearance of all parts of an object. In order to control the capacity of the model, the smoothness of the interpolator can be increased by replacing IoU with a softer version, which we do next.

Smoother Overlap Measure. The IoU measure is a special case of the following family of PD kernels:

Theorem 1. Let $R$ and $Q$ be vectors in a Hilbert $\mathcal{H}$ space such that $\langle R, R\rangle+$ $\langle Q, Q\rangle-\langle R, Q\rangle>0$. Then the function $\rho(R, Q)=\frac{\langle R, Q\rangle}{\langle R, R\rangle+\langle Q, Q\rangle-\langle R, Q\rangle}$ is a positive definite kernel.

The IoU is obtained when $R$ and $Q$ are indicator functions of the respective regions (because $\langle R, Q\rangle=\int R(x, y) Q(x, y) d x d y=|R \cap Q|$ ). This suggests a simple modification to construct a Soft IoU (SIoU) version of the latter. For a region $R=\left[x_{1}, x_{2}\right] \times\left[y_{1}, y_{2}\right]$, the indicator can be written as $R(x, y)=$ $H\left(x-x_{1}\right) H\left(x_{2}-x\right) H\left(y-y_{1}\right) H\left(y_{2}-y\right)$ where $H(z)=[z \geq 0]$ is the Heaviside step function. SIoU is obtained by replacing the indicator by the smoother function $H_{\alpha}(z)=\exp (\alpha z) /(1+\exp (\alpha z))$ instead. Note that $\mathrm{SIoU}$ is non-zero even when regions do not intersect.

Theorem 1 provides also an interpretation of the geometric embedding $\phi^{g}$ of Eq. (2) as a vector of region coordinates relative to the anchors. In fact, its entries can be written as $\rho\left(R, R_{p_{k}, \mathbf{x}}\right)=\left\langle\psi_{\mathrm{SIoU}}(R), \psi_{\mathrm{SIoU}}\left(R_{p_{k}, \mathbf{x}}\right)\right\rangle$ where $\psi_{\mathrm{SIoU}}(R) \in$ $\mathcal{H}_{\text {SIoU }}$ is the linear embedding (feature map) induced by the kernel $\rho^{1}$.

Modeling Multiple Aspects. So far, we have assumed that all parts are always visible; however, anchors also provide a mechanism to deal with the multiple aspects of 3D objects. As depicted in Fig. 3c, as the object rotates out of plane, anchors naturally appear and disappear, therefore activating and de-activating

${ }^{1}$ The anchor vectors $\psi_{\text {SIoU }}\left(R_{p_{k}, \mathbf{x}}\right)$ are not necessarily orthonormal (they are if anchors do not overlap), but this can be restored up to a linear transformation of the coordinates. 
aspect-specific components in the model. In turn, this allows to model viewpointspecific parts or appearances. In practice, we extract the $L$ highest scoring detections $R_{l}$ of the same anchor $p_{k}$, and keep the one closest to $R$.

In order to allow anchors to turn off in the model, the geometric embedding is modified as follows. Let $s_{k}\left(R_{l} \mid \mathbf{x}\right)$ be the detection score of anchor $k$ in correspondence of the region $R_{l}$; then

$$
\rho\left(R, R_{p_{k}, \mathbf{x}}\right)=\max _{l \in\{1, \ldots, L\}} \operatorname{SIoU}\left(R, R_{l}\right) \times \max \left\{0, s_{k}\left(R_{l} \mid \mathbf{x}\right)\right\} .
$$

If the anchor is never detected $\left(s_{k}\left(R_{l} \mid \mathbf{x}\right) \leq 0\right.$ for all $\left.R_{l}\right)$ then $\rho\left(R, R_{p_{k}, \mathbf{x}}\right)=0$. Furthermore, this expression also disambiguates ambiguous anchor detections by picking the one closest to $R$. Note that in Eq. (4) one can still interpret the factors $\operatorname{SIoU}\left(R, R_{l}\right)$ as projections $\left\langle\psi_{\mathrm{SIoU}}(R), \psi_{\mathrm{SIoU}}\left(R_{l}\right)\right\rangle$.

Relation to DPM. DPM is also a MIL method using a joint embedding $\phi^{\mathrm{DPM}}\left(\mathbf{x} \mid R_{1}, \ldots, R_{K}\right)$ that codes simultaneously for the appearance of $K$ parts and their pairwise geometric relationships. Our Webly-supervised learning problem requires a representation that can bridge object-focused images (where several parts are visible together as components) and part-focused images (where parts are regarded as objects in their own right). This is afforded by our embedding $\phi^{a g}(\mathbf{x} \mid R)$ but not by the DPM one. Besides bridging parts as components and parts as objects, our embedding is very robust (important in order to deal with very noisy training labels), automatically codes for multiple object aspects, and bridges unsupervised non-semantic parts (the anchors) with semantic ones.

\subsection{Anchors: Weakly-Supervised Non-semantic Parts}

The geometric embedding in the previous section leverages the power of an intermediate representation: a collection of anchors $\left\{p_{k}\right\}_{k=1}^{K}$, learned automatically using weak supervision. While there are many methods to discover discriminative non-semantic mid-level parts from image collections (Sect.1.1), here we propose a simple alternative that, empirically, works better in our context.

We learn the anchors using a formulation similar to the MIL objective (Eq. (1)):

$$
\min _{\boldsymbol{\omega}_{1}, \ldots, \boldsymbol{\omega}_{K}} \sum_{k=1}^{K}\left[\frac{\lambda}{2}\left\|\boldsymbol{\omega}_{k}\right\|^{2}-\frac{1}{n} \sum_{i=1}^{n} y_{i}\left[\max _{R \in \mathcal{R}\left(\mathbf{x}_{i}\right)}\left\langle\phi^{a}\left(\mathbf{x}_{i} \mid R\right), \boldsymbol{\omega}_{k}\right\rangle\right]_{+}\right]+\gamma \sum_{k \neq q}\left\langle\frac{\boldsymbol{\omega}_{k}}{\left\|\boldsymbol{\omega}_{k}\right\|}, \frac{\boldsymbol{\omega}_{q}}{\left\|\boldsymbol{\omega}_{q}\right\|}\right\rangle^{2},
$$

where $[z]_{+}=\max \{0, z\}$. Intuitively, anchors are learnt as discriminative midlevel parts using weak supervision. Anchor scores $s_{k}(R \mid \mathbf{x})=\left\langle\phi^{a}(\mathbf{x} \mid R), \boldsymbol{\omega}_{k}\right\rangle$ are parametrized by vectors $\boldsymbol{\omega}_{1}, \ldots, \boldsymbol{\omega}_{K}$; the first term in Eq. (5) is akin to the baseline MIL formulation of Sect.2.1 and encourages each anchor $p_{k}$ to score highly in images $\mathbf{x}_{i}$ that contain the object $\left(y_{i}=+1\right)$ and to be inactive otherwise $\left(y_{i}=-1\right)$. The last term is very important and encourages the learned models $\left\{\boldsymbol{\omega}_{k}\right\}_{k=1}^{K}$ to be mutually orthogonal, enforcing diversity. Note that anchors use the pure appearance-based region descriptor $\phi^{a}(\mathbf{x})$ since the geometric-aware 
descriptor $\phi^{a g}(\mathbf{x})$ can be computed only once anchors are available. Optimization uses stochastic gradient descent with momentum.

This formulation is similar to the MIL approach of [45] which, however, does not contain the orthogonality term. When this term is removed, we observed that the solution degenerates to detecting the most prominent object in an image. [39] uses instead a significantly more complex formulation inspired by mode seeking; in practice we opted for our approach due to its simplicity and effectiveness.

\subsection{Incorporating Strong Annotations in MIL}

While we are primarily interested in understanding whether semantic object parts can be learned from Web sources alone, in some cases the precise definition of the extent of a part is inherently ambiguous (e.g. what is the extent of a "human nose"?). Different benchmark datasets may use somewhat different definition of these concepts, making evaluation difficult. In order to remove or at least reduce this dataset-dependent ambiguity, we also explore the idea of using a single strongly annotated example to fix this degree of freedom.

Denote by $\left(\mathbf{x}_{a}, R_{a}\right)$ the single strongly-annotated example of the target part. This is incorporated in the MIL formulation, Eq. (1), by augmenting the score with a factor that compares the appearance of a region to that of $R_{a}$ :

$$
\left\langle\phi\left(\mathbf{x}_{i} \mid R\right), \mathbf{w}\right\rangle \times \begin{cases}\frac{1}{C} \exp \beta\left\langle\phi^{a}\left(\mathbf{x}_{i} \mid R\right), \phi^{a}\left(\mathbf{x}_{a} \mid R_{a}\right)\right\rangle, & y_{i}=+1 \\ 1, & y_{i}=-1\end{cases}
$$

where $C=\operatorname{avg}_{i: y_{i}=+1} \exp \beta\left\langle\phi^{a}\left(\mathbf{x}_{i} \mid R\right), \phi^{a}\left(\mathbf{x}_{a} \mid R_{a}\right)\right\rangle$ is a normalizing constant. In practice, this is used only during re-localization rounds of the training phase to guide spatial selection; at test time, bounding boxes are scored solely by the model of Eq. (1) without the additional term. Other formulations, that may use a mixture of strongly and Webly supervised examples, are also possible. However, this is besides our focus, which is to see whether parts are learnable from the Web automatically, and the single supervision is only meant to reduce the ambiguity in the task for evaluation.

\section{$3 \quad$ Experiments}

This section thoroughly evaluates the proposed method. Our main evaluation is a comparison with existing state-of-the-art techniques on the task of Weblysupervised semantic part learning. In Sect.3.1 we show that our method is substantially more accurate than existing alternatives and, in some cases, close to fully-supervised part learning.

Having established that, we then evaluate the weakly-supervised mid-level part learning (Sect.2.2) that is an essential part of our approach. It compares favorably in terms of simplicity, scalability, and accuracy against existing alternatives for discriminability as well as spatial matching of object categories (Sect. 3.2). 
Datasets. The Labeled Face Parts in the Wild (LFPW) dataset [51] contains about 1200 face images annotated with outlines for landmarks. Outlines are converted into bounding box annotations and images with missing annotations are removed from the test set. These test images are used to locate the following entities: face, eye, eyebrow, nose, and mouth.

The PascalParts dataset [47] augments the PASCAL VOC 2010 dataset with segmentation masks for object parts. Segmentation masks are converted into bounding boxes for evaluation. Parts of the same type (e.g. left and right wheels) are merged in a single entity (wheel). Objects marked as truncated or difficult are not considered for evaluation. The evaluation focuses on the bus and car categories with 18 entity types overall: car, bus, and their door, front, headlight, mirror, rear, side, wheel, and window parts. This dataset is more challenging, as entities have large intra-class appearance and pose variations. The evaluation is performed on images from the validation set that contain at least one object instance. Furthermore, following [48], object occurrences are roughly localized before detecting the parts using their localization procedure. Finally, objects whose bounding box larger side is smaller than 80 pixels are removed as several parts are nearly invisible below that scale.

The training sets from both datasets are utilized solely for training the fully supervised baselines (Sect. 3.1), and they are not used by MIL approaches.

Experimental Details. Regions are extracted using selective search [50], and described using $\ell_{2}$-normalized Decaf [52] fc6 features to compute the appearance embedding $\phi^{a}(\mathbf{x} \mid R)$. The context descriptor $\mu(R)$ is extracted from a region triple the size of $R$. The joint appearance-geometric embedding $\phi^{a g}(\mathbf{x} \mid R)$ is obtained by first extracting the top $L=5$ non-overlapping detections of each anchor and then applying Eqs. (3) and (4).

A separate mid-level anchor dictionary $\left\{p_{1}, \ldots, p_{K}\right\}$ is learnt for each object class using the Web images for all the semantic parts for the target object (including images of the object as a whole) as positive images and the background clutter images of [53] as negative ones. Equation (5) is optimized using stochastic gradient descend (SGD) with momentum for 40k iterations, alternating between positive and negative images. We train 150 anchor detectors per object class.

MIL semantic part detectors are trained solely on the Web images and the background class of [53] is used as negative bag for all the objects. The first five relocalization rounds are performed using the appearance only and the following five use the joint appearance-geometry descriptor (the joint embedding performs better with these two distinct steps). The MIL $\lambda$ hyperparameter is set by performing leave-one-category-out cross-validation.

Web images for parts are acquired by querying the BING image search engine. For car and bus parts, the query concatenates the object and the part names (e.g. "car door"). For face parts, we do not use the object name. We retrieve 500 images of the class corresponding to the object itself and 100 images of all other semantic part classes. 


\subsection{Webly Supervised Localization of Objects and Semantic Parts}

This section evaluates the detection performance of our approach. We gradually incorporate the proposed improvements, i.e. the context descriptor $(\mathrm{C})$ and the geometrical embedding $(\mathrm{G})$ to the basic MIL baseline (B) as defined in Sect. 2.1 and monitor their impact.

We compare our method to the state-of-the-art co-localization algorithm of Cho et al. [25] and the state-of-the-art weakly supervised detection method from Bilen and Vedaldi [24]. To detect a given part with [25], we run their code on all images that contain that part (e.g. for co-localizing eyes we consider face and eye images). As reference, we also report a fully supervised detector, trained using bounding-boxes from the training set, for all objects and parts (F). For this, we use the R-CNN method of [54] on top of the same features used in MIL.

We mainly report the Average Precision (AP) per part/object class and its average (mAP) over all parts in each class. We also report the CorLoc (for correct localization) measure, as it is often used in the co-localization literature $[14,55]$. As most parts in both datasets are relatively small, following [47], the IoU threshold for correct detection is set to 0.4 .

Table 1. Part detection results averaged for the face, car, and bus parent classes. $\mathrm{mAP}$ and average CorLoc for the MIL baseline (B), our improved versions that use context $(\mathrm{C})$, geometrical embedding $(\mathrm{G})$ compared to the fully supervised R-CNN $(\mathrm{F})$.

\begin{tabular}{l|l|l|l|l|l|c}
\hline Measure & \multicolumn{2}{l}{ mAP } & \multicolumn{3}{l}{ averageCorLoc } \\
\hline Parent class & $\{$ Face $\}$ & $\{$ Car $\}$ & $\{$ Bus $\}$ & $\{$ Face $\}$ & $\{$ Car $\}$ & $\{$ Bus $\}$ \\
\hline Cho et al. $[25]$ & 16.6 & 16.9 & 12.4 & 31.4 & 29.9 & 15.5 \\
\hline Bilen and Vedaldi $[24]$ & 2.7 & 12.0 & 4.7 & 7.2 & 15.3 & 6.7 \\
\hline B & 20.6 & 29.1 & 22.7 & 22.0 & 38.1 & 29.4 \\
\hline B+C & 22.4 & 27.3 & 21.4 & 29.1 & 37.6 & 28.4 \\
\hline B+G & 29.0 & 34.1 & $\mathbf{2 3 . 3}$ & 33.1 & 45.5 & $\mathbf{3 1 . 5}$ \\
\hline B+C+G & $\mathbf{4 4 . 9}$ & $\mathbf{3 4 . 4}$ & 23.0 & $\mathbf{5 2 . 5}$ & $\mathbf{4 7 . 8}$ & 29.6 \\
\hline F & 53.7 & 51.2 & 48.2 & 60.5 & 62.9 & 63.8 \\
\hline F+C+G & $\mathbf{6 1 . 4}$ & $\mathbf{6 0 . 3}$ & $\mathbf{5 4 . 1}$ & $\mathbf{6 7 . 8}$ & $\mathbf{7 1 . 8}$ & $\mathbf{6 6 . 0}$ \\
\hline
\end{tabular}

Results. Table 1 reports the average AP and CorLoc over all parts of a given object class for all these methods. First, we observe that even the MIL baseline (B) outperforms off-the-shelf methods such as [24,25]. For [24], we have observed that the part detectors degrade to detecting subparts of semantic parts, suggesting that [24] lacks robustness to drastic scale variations and to the large amount of noise present in our dataset. Second, we see that using the geometric embedding $(+\mathrm{G})$ always improves the baseline results by $1-10 \mathrm{mAP}$ points. On top of geometry, using context $(+\mathrm{C})$ helps for face and car parts, but not for buses. Overall the unified embedding brings a large improvement for faces $(+24.3 \mathrm{mAP})$ 
and for cars (+5.3 mAP) and more contained for buses (+0.6 mAP). Importantly, these improvements significantly reduce the gap between using noisy Web supervision and the fully supervised R-CNN (F); overall, Webly supervision achieves respectively $84 \%, 67 \%$, and $48 \%$ of the performance of $(\mathrm{F})$.

Last but not least, we also experimented extending the fully supervised R-CNN method with the joint appearance-geometry embedding and context descriptor $(\mathrm{F}+\mathrm{C}+\mathrm{G})$, which improves part detections by $+7.7,+9.1,+5.9 \mathrm{mAP}$ points respectively. This suggests that our representation may be applicable well beyond weakly supervised learning.

Table 2. Individual part detection results for car: APs for the MIL baseline (B), our improved versions that use context (C), geometrical embedding (G) and the different flavors of the fully supervised R-CNN (F).

\begin{tabular}{l|l|c|l|l|c|l|l|l|l|l|l}
\hline \multicolumn{1}{l|}{ Class } & Door & Rear & Wheel & Wind & Side & Car & Front & Headl & Mirror & Mean $\{$ car $\}$ \\
\hline \multirow{2}{*}{ Web } & B & 0.4 & 10.8 & 34.9 & 3.6 & 63.1 & 92.6 & $\mathbf{5 5 . 2}$ & 0.7 & $\mathbf{0 . 3}$ & 29.1 \\
\cline { 2 - 12 } & B+C & 0.8 & 11.4 & 31.3 & 4.9 & 58.8 & 83.0 & 54.0 & $\mathbf{1 . 0}$ & 0.2 & 27.3 \\
\cline { 2 - 12 } & B+G & 0.7 & 11.8 & $\mathbf{4 7 . 9}$ & $\mathbf{2 2 . 7}$ & 71.3 & $\mathbf{9 7 . 8}$ & 54.5 & 0.2 & 0.2 & 34.1 \\
\cline { 2 - 11 } & B+C+G & $\mathbf{5 . 1}$ & $\mathbf{1 4 . 7}$ & 43.6 & 22.6 & $\mathbf{7 2 . 3}$ & 95.7 & 54.7 & 0.3 & 0.2 & $\mathbf{3 4 . 4}$ \\
\hline \multirow{2}{*}{ Full } & F & 17.0 & $\mathbf{3 9 . 0}$ & 66.3 & 53.3 & 83.2 & 95.1 & 75.9 & 25.3 & 5.5 & 51.2 \\
\cline { 2 - 10 } & F+C+G & $\mathbf{3 1 . 1}$ & 30.7 & $\mathbf{7 2 . 3}$ & $\mathbf{6 7 . 3}$ & $\mathbf{9 0 . 1}$ & $\mathbf{9 8 . 7}$ & $\mathbf{8 2 . 9}$ & $\mathbf{4 8 . 1}$ & $\mathbf{2 1 . 3}$ & $\mathbf{6 0 . 3}$ \\
\hline
\end{tabular}

Table 2 shows per-part detection results for the car parts. We see that geometry helps for 6 parts out of 9 . Out of the three remaining parts, two are cases where the MIL baseline failed. In the less ambiguous fully-supervised scenario, the geometric embedding improves the performance in 8 out of 9 cases.

Leveraging a Single Annotation. As noted in Sect. 2.3, one issue with weakly supervised part learning is the inherent ambiguity in the part extent, that may differ from dataset to dataset. Here we address the ambiguity by adding a single strong annotation to the mix using the method described in Sect. 2.3. We asked an annotator to select 25 representative part annotations per part class from the training sets of each dataset. We retrain every part detector for each of the annotations and report mean and standard deviation of mAP. As a baseline, we also consider an exemplar detector trained using the single annotated example (A).

Results are reported in Table 3. Compared to pure Web supervision $(\mathrm{B}+\mathrm{C}+\mathrm{G})$ in Table 1 , the single annotation $(\mathrm{A}+\mathrm{B}+\mathrm{C}+\mathrm{G})$ does not help for faces, for which the proposed method was already working very well, but there is a $+2 \mathrm{mAP}$ point improvement for cars and $+6.8 \mathrm{mAP}$ for buses, which are more challenging. We also note that the complete method $(\mathrm{A}+\mathrm{B}+\mathrm{C}+\mathrm{G})$ is substantially superior to the exemplar detector (A). 
Table 3. Part detection results using a single strong annotation (A): mAP and average CorLoc for the MIL baseline (B), our improved versions that use context (C), geometrical embedding $(\mathrm{G})$. Mean and standard deviation over 25 random annotations.

\begin{tabular}{l|l|l|l|l|l|l}
\hline Measure & mAP & \multicolumn{5}{l}{ averageCorLoc } \\
\hline Parent class & $\{$ Face $\}$ & $\{$ Car $\}$ & $\{$ Bus $\}$ & $\{$ Face $\}$ & $\{$ Car $\}$ & $\{$ Bus $\}$ \\
\hline $\mathrm{A}$ & $29.4 \pm 2.6$ & $25.1 \pm 2.7$ & $24.5 \pm 2.7$ & $38.2 \pm 2.5$ & $39.8 \pm 3.2$ & $39.6 \pm 3.2$ \\
\hline $\mathrm{A}+\mathrm{B}$ & $27.3 \pm 3.1$ & $33.3 \pm 1.1$ & $26.9 \pm 1.3$ & $34.6 \pm 3.7$ & $46.6 \pm 1.5$ & $40.0 \pm 2.3$ \\
\hline $\mathrm{A}+\mathrm{B}+\mathrm{C}$ & $38.2 \pm 3.1$ & $32.4 \pm 1.2$ & $26.6 \pm 1.6$ & $51.7 \pm 3.2$ & $49.4 \pm 1.5$ & $43.9 \pm 3.0$ \\
\hline $\mathrm{A}+\mathrm{B}+\mathrm{G}$ & $34.5 \pm 4.3$ & $35.7 \pm 1.1$ & $28.1 \pm 1.2$ & $43.5 \pm 4.8$ & $48.8 \pm 1.6$ & $42.2 \pm 2.2$ \\
\hline $\mathrm{A}+\mathrm{B}+\mathrm{C}+\mathrm{G}$ & $\mathbf{4 3 . 0} \pm \mathbf{3 . 6}$ & $\mathbf{3 6 . 4} \pm \mathbf{1 . 0}$ & $\mathbf{3 0 . 1} \pm \mathbf{1 . 8}$ & $\mathbf{5 4 . 7} \pm \mathbf{3 . 2}$ & $\mathbf{5 1 . 6} \pm \mathbf{1 . 6}$ & $\mathbf{4 5 . 9} \pm \mathbf{2 . 8}$ \\
\hline
\end{tabular}

\subsection{Validation of Weakly-Supervised Mid-level Anchors}

This section validates the mid-level anchors (Sect.2.2) against alternatives in terms of discriminative information content and its ability of establishing meaningful matches between images, which is a key requirement in our application.

Discriminative Power of Anchors. Since most of the existing methods for learning mid-level patches are evaluated in terms of discriminative content in a classification setting, we adopt the same protocol here. In particular, we evaluate the anchors as mid-level patches on the MIT Scene 67 indoor scene classification task [56]. The pipeline first learns 50 mid-level anchors for each of the 67 scene classes. Then, similar to [43], images are split into spatial grids $(2 \times 2$ and $1 \times 1)$ and described by concatenating the maximum scores attained by each anchor detector inside each bin of the grid. All the grid descriptors are then concatenated to form a global image descriptor which is $\ell_{2}$ normalized. 67 one-vs-rest SVM classifiers are trained on top of these descriptors. To be comparable with other methods, we consider both Decaf fc6 and VGG-VD fc7 [57] descriptors.

Table 4 contains the results of the classification experiment. Our weaklysupervised anchors clearly outperform other mid-level element approaches that are not based on CNN features [39,40,44,45]. Among CNN based approaches, our method outperforms the state-of-the-art mid-level feature based method from [43] on both VGG-VD and Decaf features. Remarkably, using our part detectors

Table 4. Classification results on MIT scenes [56]. Methods using mid-level elements are marked with ${ }^{\dagger}$. For CNN-based approaches, features rely on Decaf or VGG-VD.

\begin{tabular}{l|l|l|l|l|l|l}
\hline Method & $\mathrm{BoP}^{\dagger}[40]$ & $\begin{array}{l}\mathrm{DMS}^{\dagger} \\
{[39]}\end{array}$ & $\begin{array}{l}\text { Jian et al. } \\
{[45]}\end{array}$ & $\begin{array}{l}\mathrm{RFDC}^{\dagger} \\
{[44]}\end{array}$ & $\begin{array}{l}\text { FC Decaf } \\
{[52]}\end{array}$ & $\begin{array}{l}\text { FC VGG-VD } \\
{[57]}\end{array}$ \\
\hline Accuracy (\%) & 46.1 & 64.0 & 58.1 & 54.4 & 57.7 & 68.9 \\
\hline \hline \multirow{2}{*}{ Method } & $\begin{array}{l}\mathrm{BoE}^{\dagger} \\
\text { Decaf } \\
{[43]}\end{array}$ & $\begin{array}{l}\text { ours } \\
\text { Decaf }\end{array}$ & $\begin{array}{l}\text { FV-CNN } \\
\text { Decaf }[58]\end{array}$ & $\begin{array}{l}\text { BoE }^{\dagger} \\
V G G-V D \\
{[43]}\end{array}$ & $\begin{array}{l}\text { ours }^{\dagger} \\
V G G-V D\end{array}$ & $\begin{array}{l}\text { FV-CNN } \\
V G G-V D \\
{[58]}\end{array}$ \\
\hline Accuracy (\%) & 69.7 & $\mathbf{7 1 . 5}$ & 69.7 & 77.6 & 77.8 & $\mathbf{8 1 . 6}$ \\
\hline
\end{tabular}


improves over the baseline which uses the global image CNN descriptor (FC) by 13.8 and 8.7 average accuracy points for Decaf and VGG-VD features respectively. Compared to other methods which are not based on detecting mid-level elements, our pipeline outperforms state-of-the-art FV-CNN for Decaf features and is inferior for VGG-VD.

Ability of Anchors to Establish Semantic Matches. The previous experiment assessed favorably the mid-level parts in terms of discriminative content; however, in the embedding $\phi^{g}$, these are used as geometric anchors. Hence, here we validate the ability of the mid-level anchors to induce good semantic matches between pairs of images (before learning the semantic part models).

To perform semantic matching between a source image $x_{S}$ and a target image $x_{T}$, we consider each part annotation $R_{S}$ in the source and predict its best match $\hat{R}_{T}$ in the target. The quality of the match is evaluated by measuring the IoU between the predicted $\hat{R}_{T}$ and ground-truth $R_{T}$ part. When a part appears more than once (e.g. eyes often appear twice), we choose the most overlaping pair. Performance is reported by averaging the match IoU for all part occurrences and pairs of images in the test set, reporting the results for each object category.

Given a source part $R_{S}$, the joint appearance-geometry embedding (anchor-ag) is extracted for the source part $\phi^{a g}\left(\mathbf{x}_{S} \mid R_{S}\right)$ and the target region $\hat{R}_{T}$ that maximizes the inner product $\left\langle\phi^{a g}\left(\mathbf{x}_{S} \mid R_{S}\right), \phi^{a g}\left(\mathbf{x}_{T} \mid \hat{R}_{T}\right)\right\rangle$ is returned as the predicted match. We also compare anchor-g that uses only the geometric embedding $\phi^{g}(\mathbf{x} \mid R)$ and the baseline $a$ that uses only the appearance embedding $\phi^{a}(\mathbf{x} \mid R)$.

We also compare two strong off-the-shelf baselines: DSP [59], state-of-the-art pairwise semantic matching method, and the method of [60], state-of-the-art for joint alignment. To perform box matching with [59] and [60] we fit an affine transformation to the disparity map contained inside a given source bounding box and apply this transform to move this box to the target image. Due to

Table 5. Semantic matching. For every parent class, we report average overlap (IoU) over all semantic parts. The face class results are obtained on the LFPW dataset while bus and car results come from the PascalParts dataset.

\begin{tabular}{l|l|l|l|l|l|l}
\hline \multirow{2}{*}{ Set } & Parent class & \multicolumn{4}{l}{ Matching method } \\
\cline { 3 - 7 } & & Anchor-ag & Anchor-g & $\mathrm{a}$ & Flowweb [60] & DSP [59] \\
\hline \multirow{3}{*}{50 images } & $\{$ Car $\}$ & $\mathbf{0 . 3 6}$ & $\mathbf{0 . 3 6}$ & 0.31 & 0.34 & 0.23 \\
\cline { 2 - 7 } & $\{$ Bus $\}$ & $\mathbf{0 . 3 7}$ & 0.36 & 0.31 & 0.31 & 0.22 \\
\cline { 2 - 7 } & $\{$ Face & 0.41 & 0.39 & 0.33 & $\mathbf{0 . 4 3}$ & 0.19 \\
\hline \multirow{5}{*}{ Full } & $\{$ Car $\}$ & $\mathbf{0 . 3 6}$ & $\mathbf{0 . 3 6}$ & 0.30 & - & 0.22 \\
\cline { 2 - 7 } & $\{$ Bus $\}$ & $\mathbf{0 . 3 5}$ & $\mathbf{0 . 3 5}$ & 0.29 & - & 0.21 \\
\cline { 2 - 7 } & $\{$ Face $\}$ & $\mathbf{0 . 4 1}$ & 0.39 & 0.34 & - & 0.21 \\
\hline
\end{tabular}


scalability issues, we were unable to apply [60] to the full dataset ${ }^{2}$, so we perform this comparison on a random subset of 50 images.

Table 5 presents the results of our benchmark. On the small subset of 50 images the costly approach of [60] performs better than our embedding only on the LFPW faces, where the viewpoint variation is limited. On the car and bus categories our method outperforms [60] by $10 \%$ and $16 \%$ average IoU respec-

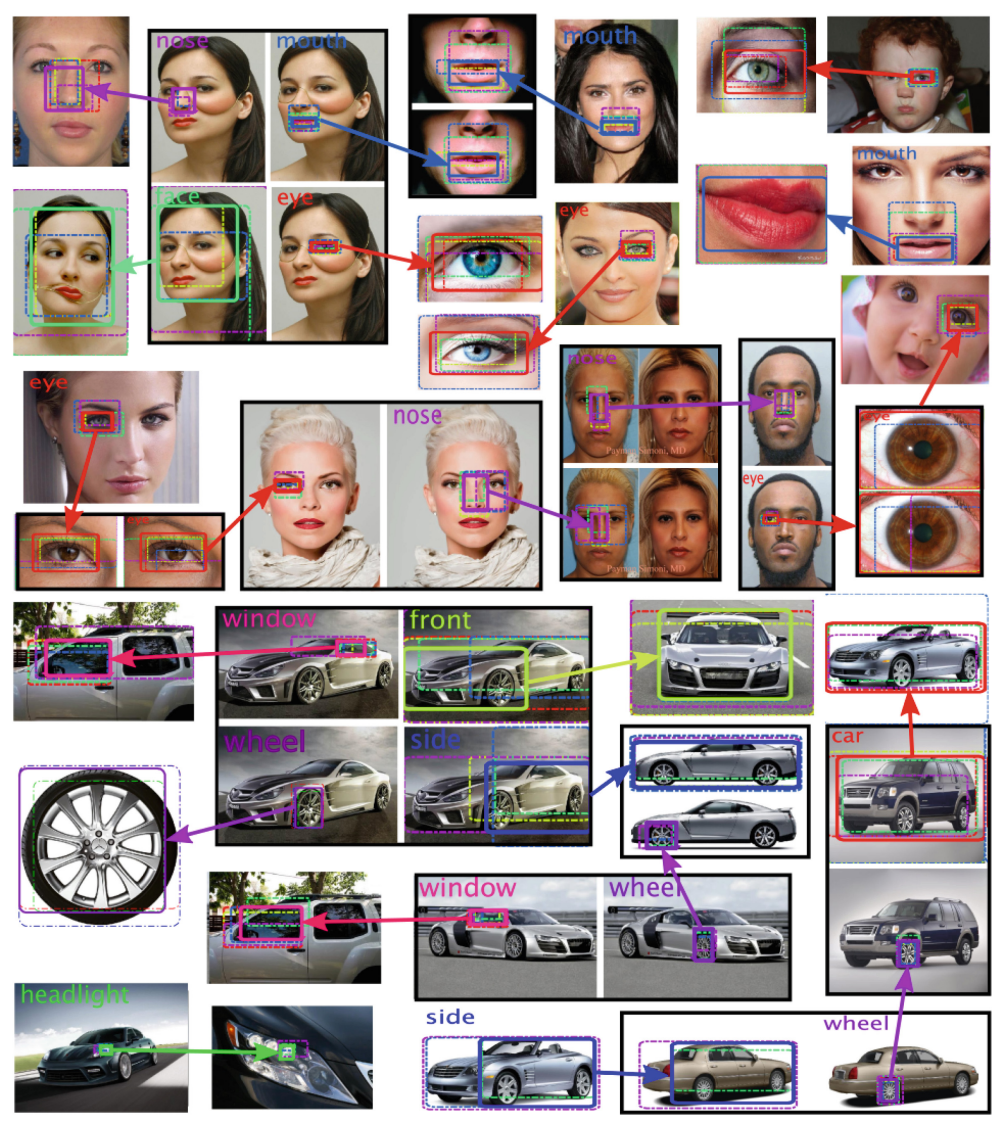

Fig. 4. Navigating the visual semantic atlas. Each pair of solid bounding boxes connected by an arrow denotes a preselected part bounding box (near the starting point of an arrow) as detected by our algorithm and the most similar semantic match (the endpoint of the arrow). The best matching bounding box is the detection with highest appearance-geometry descriptor similarity among all the detections in our database of web images. The dashed boxes denote anchors that contributed the most to the similarity. Please note that the matching gracefully occurs across scales.

\footnotetext{
${ }^{2}$ More precisely, we were not able to apply [60] on a dataset with more than $60128 \times 68$
} pixel images on a server with 120 GB of RAM. 
tively. Our method is also consistently better than DSP [59], on both the small and full test set.

We also note that the matching using geometric embeddings alone (anchor-g) achieves similar performance than the appearance-geometry matching (anchor$a g)$ which validates our intuition that the local geometry of an object is wellcaptured by the anchors.

\section{An Atlas for Visual Semantic}

As a byproduct of Webly-supervised learning, our method annotates the Web images with semantic parts. By endowing an image dataset with such concepts, we show here that it is possible to browse these annotated images. All of this composes our visual semantic atlas (see a subset of the atlas in Fig. 4) that allows to navigate from one image to another, even between an image of a full object and a zoomed-in image of one of its parts.

\section{Conclusions}

We have proposed a novel method for learning about objects, their semantic parts, and their geometric relationships, from noisy Web supervision. This is achieved by first learning a weakly supervised dictionary of mid-level visual elements which define a robust object-centric coordinate frame. Such property theoretically motivates our approach. The geometric projections are then used in a novel appearance-geometry embedding that improves learning of semantic object parts from noisy Web data. We showed improved performance over co-localization [25], deep weakly supervised approach [24] and a MIL baseline on all benchmarked datasets. Extensive evaluation of our proposed mid-level elements shows comparable results to state-of-the-art in terms of their discriminative power and superior results in terms of the ability to establish semantic matches between images. Finally, our method also provides a visually intuitive way to navigate Web images and predicted annotations.

Acknowledgments. We would like to thank Xerox Research Center Europe and ERC 677195-IDIU for supporting this research.

\section{References}

1. Krizhevsky, A., Sutskever, I., Hinton, G.E.: Imagenet classification with deep convolutional neural networks. In: Proceeding of NIPS (2012)

2. Frome, A., Corrado, G.S., Shlens, J., Bengio, S., Dean, J., Ranzato, M.A., Mikolov, T.: Devise: a deep visual-semantic embedding model. In: Proceeding of NIPS (2013)

3. Karpathy, A., Joulin, A., Fei-Fei, L.: Deep fragment embeddings for bidirectional image-sentence mapping. In: Proceeding of NIPS (2014)

4. Simonyan, K., Zisserman, A.: Two-stream convolutional networks for action recognition in videos. In: Proceeding of NIPS (2014) 
5. Russakovsky, O., et al.: Imagenet large scale visual recognition challenge. Int. J. Comput. Vis. 115(3), 211-252 (2015)

6. Deng, J., Dong, W., Socher, R., Li, L.J., Li, K., Fei-Fei, L.: ImageNet: a large-scale hierarchical image database. In: Proceeding of CVPR (2009)

7. Nguyen, M.H., Torresani, L., de la Torre, F., Rother, C.: Weakly supervised discriminative localization and classification: a joint learning process. In: Proceeding of ICCV (2009)

8. Pandey, M., Lazebnik, S.: Scene recognition and weakly supervised object localization with deformable part-based models. In: Proceeding of ICCV (2011)

9. Deselaers, T., Alexe, B., Ferrari, V.: Weakly supervised localization and learning with generic knowledge. Int. J. Comput. Vis. 100(3), 275-293 (2012)

10. Wang, C., Ren, W., Huang, K., Tan, T.: Weakly supervised object localization with latent category learning. In: Fleet, D., Pajdla, T., Schiele, B., Tuytelaars, T. (eds.) ECCV 2014. LNCS, vol. 8694, pp. 431-445. Springer, Heidelberg (2014). doi:10.1007/978-3-319-10599-4_28

11. Hoffman, J., Guadarrama, S., Tzeng, E.S., Hu, R., Donahue, J., Girshick, R., Darrell, T., Saenko, K.: LSDA: large scale detection through adaptation. In: Ghahramani, Z., Welling, M., Cortes, C., Lawrence, N., Weinberger, K., eds.: Proceeding of NIPS (2014)

12. Hoffman, J., Pathak, D., Darrell, T., Saenko, K.: Detector discovery in the wild: joint multiple instance and representation learning. In: Proceeding of CVPR (2015)

13. Cinbis, R.G., Verbeek, J., Schmid, C.: Weakly supervised object localization with multi-fold multiple instance learning. In: PAMI, September 2015

14. Joulin, A., Tang, K., Fei-Fei, L.: Efficient image and video co-localization with Frank-Wolfe algorithm. In: Fleet, D., Pajdla, T., Schiele, B., Tuytelaars, T. (eds.) ECCV 2014. LNCS, vol. 8694, pp. 253-268. Springer, Heidelberg (2014). doi:10. 1007/978-3-319-10599-4_17

15. Tang, K., Joulin, A., Li, L.J., Fei-Fei, L.: Co-localization in real-world images. In: Proceeding of CVPR (2014)

16. Ali, K., Saenko, K.: Confidence-rated multiple instance boosting for object detection. In: Proceeding of CVPR (2014)

17. Shi, Z., Hospedales, T., Xiang, T.: Bayesian joint modelling for object localisation in weakly labelled images. PAMI 37(10), 1959-1972 (2015)

18. Joulin, A., Bach, F., Ponce, J.: Efficient optimization for discriminative latent class models. In: Proceeding of NIPS (2010)

19. Vicente, S., Rother, C., Kolmogorov, V.: Object cosegmentation. In: Proceeding of CVPR (2011)

20. Joulin, A., Bach, F., Ponce, J.: Multi-class cosegmentation. In: Proceeding of CVPR (2012)

21. Rubinstein, M., Joulin, A., Kopf, J., Liu, C.: Unsupervised joint object discovery and segmentation in internet images. In: Proceeding of CVPR (2013)

22. Song, H.O., Girshick, R., Jegelka, S., Mairal, J., Harchaoui, Z., Darrell, T.: On learning to localize objects with minimal supervision. In: Proceeding of ICML (2014)

23. Li, Q., Wu, J., Tu, Z.: Harvesting mid-level visual concepts from large-scale internet images. In: Proceeding of CVPR (2013)

24. Bilen, H., Vedaldi, A.: Weakly supervised deep detection networks. arXiv preprint (2015). arXiv:1511.02853

25. Cho, M., Kwak, S., Schmid, C., Ponce, J.: Unsupervised object discovery and localization in the wild: part-based matching with bottom-up region proposals. In: Proceeding of CVPR (2015) 
26. Fergus, R., Fei-Fei, L., Perona, P., Zisserman, A.: Learning object categories from google's image search. In: Proceeding of ICCV, pp. 1816-1823 (2005)

27. Parkhi, O.M., Vedaldi, A., Zisserman, A.: On-the-fly specific person retrieval. In: International Workshop on Image Analysis for Multimedia Interactive Services. IEEE (2012)

28. Schroff, F., Criminisi, A., Zisserman, A.: Harvesting image databases from the web. In: Proceeding of ICCV (2007)

29. Tsai, D., Jing, Y., Liu, Y., Rowley, H., Ioffe, S., Rehg, J.: Large-scale image annotation using visual synset. In: Proceeding of ICCV, pp. 611-618 (2011)

30. Kim, G., Xing, E.P.: On Multiple Foreground cosegmentation. In: Proceeding of CVPR (2012)

31. Chen, X., Gupta, A.: Webly supervised learning of convolutional networks. In: Proceeding of ICCV (2015)

32. Chen, X., Shrivastava, A., Gupta, A.: Neil: extracting visual knowledge from web data. In: Proceeding of ICCV (2013)

33. Divvala, S.K., Farhadi, A., Guestrin, C.: Learning everything about anything: webly-supervised visual concept learning. In: Proceeding of CVPR (2014)

34. Felzenszwalb, P.F., Huttenlocher, D.P.: Pictorial structures for object recognition. IJCV 61, 55-79 (2005)

35. Fergus, R., Perona, P., Zisserman, A.: Object class recognition by unsupervised scale-invariant learning. Proc. CVPR 2, 264-271 (2003)

36. Leibe, B., Leonardis, A., Schiele, B.: Robust object detection with interleaved categorization and segmentation. IJCV 77(1-3), 259-289 (2008)

37. Felzenszwalb, P.F., Girshick, R.B., McAllester, D., Ramanan, D.: Object detection with discriminatively trained part based models. PAMI 32(9), 1627-1645 (2010)

38. Singh, S., Gupta, A., Efros, A.A.: Unsupervised discovery of mid-level discriminative patches. In: Fitzgibbon, A., Lazebnik, S., Perona, P., Sato, S., Schmid, C. (eds.) ECCV 2012. LNCS, vol. 7573, pp. 73-86. Springer, Heidelberg (2012)

39. Doersch, C., Gupta, A., Efros, A.A.: Mid-level visual element discovery as discriminative mode seeking. In: Proceeding of NIPS (2013)

40. Juneja, M., Vedaldi, A., Jawahar, C.V., Zisserman, A.: Blocks that shout: distinctive parts for scene classification. In: Proceeding of CVPR (2013)

41. Endres, I., Shih, K.J., Jiaa, J., Hoiem, D.: Learning collections of part models for object recognition. In: Proceeding of CVPR (2013)

42. Doersch, C., Gupta, A., Efros, A.A.: Unsupervised visual representation learning by context prediction. In: Proceeding of ICCV (2015)

43. Li, Y., Liu, L., Shen, C., van den Hengel, A.: Mid-level deep pattern mining. In: 2015 IEEE Conference on Computer Vision and Pattern Recognition (CVPR), pp. 971-980. IEEE (2015)

44. Bossard, L., Guillaumin, M., Gool, L.: Food-101 - mining discriminative components with random forests. In: Fleet, D., Pajdla, T., Schiele, B., Tuytelaars, T. (eds.) ECCV 2014. LNCS, vol. 8694, pp. 446-461. Springer, Heidelberg (2014). doi:10.1007/978-3-319-10599-4_29

45. Sun, J., Ponce, J.: Learning dictionary of discriminative part detectors for image categorization and cosegmentation. Submitted to International Journal of Computer Vision, under minor revision (2015)

46. Zhang, N., Donahue, J., Girshick, R., Darrell, T.: Part-based R-CNNs for finegrained category detection. In: Fleet, D., Pajdla, T., Schiele, B., Tuytelaars, T. (eds.) ECCV 2014. LNCS, vol. 8689, pp. 834-849. Springer, Heidelberg (2014). doi:10.1007/978-3-319-10590-1_54 
47. Chen, X., Mottaghi, R., Liu, X., Fidler, S., Urtasun, R., Yuille, A.: Detect what you can: detecting and representing objects using holistic models and body parts. In: Proceeding of CVPR (2014)

48. Wang, P., Shen, X., Lin, Z.L., Cohen, S., Price, B.L., Yuille, A.L.: Joint object and part segmentation using deep learned potentials. In: Proceeding of ICCV (2015)

49. Dietterich, T.G., Lathrop, R.H., Lozano-Pérez, T.: Solving the multiple instance problem with axis-parallel rectangles. Artif. Intell. 89(1-2), 31-71 (1997)

50. Uijlings, J.R.R., et al.: Selective search for object recognition. Int. J. Comput. Vis. 104(2), 154-171 (2013)

51. Belhumeur, P.N., et al.: Localizing parts of faces using a consensus of exemplars. IEEE Trans. Pattern Anal. Mach. Intell. 35(12), 2930-2940 (2013)

52. Jia, Y., Shelhamer, E., Donahue, J., Karayev, S., Long, J., Girshick, R., Guadarrama, S., Darrell, T.: Caffe: convolutional architecture for fast feature embedding. arXiv preprint (2014). arXiv:1408.5093

53. Fei-Fei, L., Fergus, R., Perona, P.: Learning generative visual models from few training examples: an incremental bayesian approach tested on 101 object categories. Comput. Visi. Image Underst. 106(1), 59-70 (2007)

54. Girshick, R., Donahue, J., Darrell, T., Malik, J.: Rich feature hierarchies for accurate object detection and semantic segmentation. In: Proceeding of CVPR (2014)

55. Deselaers, T., Alexe, B., Ferrari, V.: Localizing objects while learning their appearance. In: Daniilidis, K., Maragos, P., Paragios, N. (eds.) ECCV 2010. LNCS, vol. 6314, pp. 452-466. Springer, Heidelberg (2010). doi:10.1007/978-3-642-15561-1_33

56. Quattoni, A., Torralba, A.: Recognizing indoor scenes. In: Proceeding of CVPR (2009)

57. Simonyan, K., Zisserman, A.: Very deep convolutional networks for large-scale image recognition (2014). arXiv:1409.1556

58. Cimpoi, M., Maji, S., Vedaldi, A.: Deep filter banks for texture recognition and segmentation. In: Proceeding of CVPR (2015)

59. Kim, J., Liu, C., Sha, F., Grauman, K.: Deformable spatial pyramid matching for fast dense correspondences. In: Proceeding of CVPR (2013)

60. Zhou, T., Jae Lee, Y., Yu, S.X., Efros, A.A.: Flowweb: Joint image set alignment by weaving consistent, pixel-wise correspondences. In: Proceeding of CVPR (2015) 